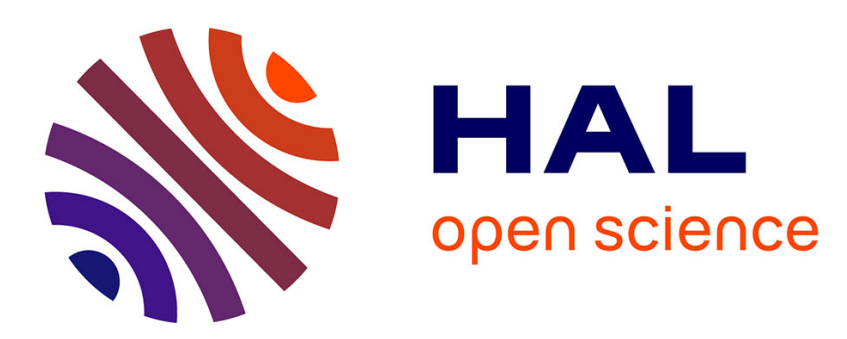

\title{
Découvertes à Saint-Aubin-sur-Mer (Calvados)
}

Eugen Eblé, Henri van Effenterre

\section{To cite this version:}

Eugen Eblé, Henri van Effenterre. Découvertes à Saint-Aubin-sur-Mer (Calvados). Gallia - Fouilles et monuments archéologiques en France métropolitaine, 1948, 6 (2), pp.365-383. 10.3406/galia.1948.2096 . hal-01920033

\section{HAL Id: hal-01920033 \\ https://hal.science/hal-01920033}

Submitted on 27 Feb 2020

HAL is a multi-disciplinary open access archive for the deposit and dissemination of scientific research documents, whether they are published or not. The documents may come from teaching and research institutions in France or abroad, or from public or private research centers.
L'archive ouverte pluridisciplinaire HAL, est destinée au dépôt et à la diffusion de documents scientifiques de niveau recherche, publiés ou non, émanant des établissements d'enseignement et de recherche français ou étrangers, des laboratoires publics ou privés.

\section{(ㅇ)(1) $\$$}

Distributed under a Creative Commons Attribution - NonCommercial - NoDerivatives 44.0 


\title{
DÉCOUVERTES A SAIN'T-AUBIN-SUR-MER
}

\author{
(Calvados)
}

\author{
par M. Eugen EBLÉ
}

[On trouvera ci-dessous un résumé des travaux effectués à Saint-Aubin-surMer (Calvados), par M. Eugen Fblé. Sur un site antique connu, mais que rien ne signalait particulièrement à l'altention des savants, M. Eblé, instituteur à Lörrach (Bade) et amateur d'archéologie, s'est trouvé pendant deux années, comme Obergefreiter de la Wermacht, chargé de suivre les travaux de fortifications du fameux mur de l'Atlantique. Il eut le mérite de recueillir et de classer les objets trouvés au hasard des terrassements, de dessiner et de photographier les murs antiques ou les tombes avant leur destruction, de relever avec soin les ćlíments des constructions dégagées. Tous ceux qui connaissent la méfiance que les archéologues inspirent parfois aux militaires apprécieront l'habileté de W. Eblé et lo service qu'il a rendu à la science. A cet égard, il fut grandement aidé du côté allemand par M. Möbius, alor's chargé de mission archéologique auprès de l'armée d'occupation et, du ròté français, par Y. Béquignon, alors Directeur de la IVe circonseription arehélogique qui fit rimpossible - dans une zone ò sa présence itait naturellement plutòt mal vue -- pour suivre la fouille et assurer la mise à labri des antiquités découvertes.

la principale trouvaille, une stalue de déesse gauloise assise, fut signalée par les deux savants dans divers comptes rendus et elle vient de faire l'objet d'une belle publication dans les. Monuments Piot ${ }^{1}$. Mais le reste de la fouille n'avait été que très sommairement esquissé et nous sommes heureux de pouvoir donner aujourd'hui, des travaux très minutieux et détaillés d'E. Eblé à Saint-Aubin, l'étude d'ensemble qui suit.

Comme on le verra, hien des points restent encore obscurs, qu'une reprise des sondages pourra seule éclaircir. Il n'a pas dépendu de l'auteur de ces lignes, ni de F. Fblé, que cette reprise n’ait été effectuée dès 1948. Le Comité des fouilles archéologiques avail, acrordé une subvention pour que des sondages fussent pratiqués avant la reconstruction des immeubles détruits durant les hostilités sur le site antique. Ie Conseil llunicipal de Saint-Aubin en avait fait autant. Hélas! les circonstances n'ont pas encore permis la reprise des travaux qui s'imposerait.

On peut espérer que les années prochaines verront cette reprise et que les nouvelles fouilles viendront en particulier apporter quelques indications sur la question qui nous semble la plus intéressante dans les études d'E. Ehlé, la persistance, au même endroit, d'un sanctuaire plusieurs fois reconstruit depuis l'époque celtique jusqu'à la conversion générale des Gaules au christianisme. Il semble par contre, à en juger par les données actuelles de la fouille, qu'il y ait rupture entre le culte et l'occupation chrétienne du site et qu'il soit difficile

(1) T. XLIII, 1949, p. 83 sq. et pl. XI. 
par conséquent de cher'her dans la déesse gauloise de Saint-Aubin, comme l'avait ingénieusement suggéré Y. Béyuignon ${ }^{1}$, une aïeule lointaine de la célèbre vierge Noire toute roisine en l'église de La Déliviande *.]

Paris, nai 1949.

Ilenri van EFfentrinkt.

Saint-Aubin-sur-Mer est une station balnéaire de la Cốte de Nacre, a $16 \mathrm{~km}$. au nord de Caen. La plage, orientie an nord, y est bordée, ver's louest, par une falaise qui surplombe de $8 \mathrm{~m}$. environ la laisse de haute mer et qui dessine une légire arancie baptiscée aujourd'hui Cap) Romain (fig. 1). La falaise s'abaisse en pente douce ver's l'arrière-pą̣s et devient

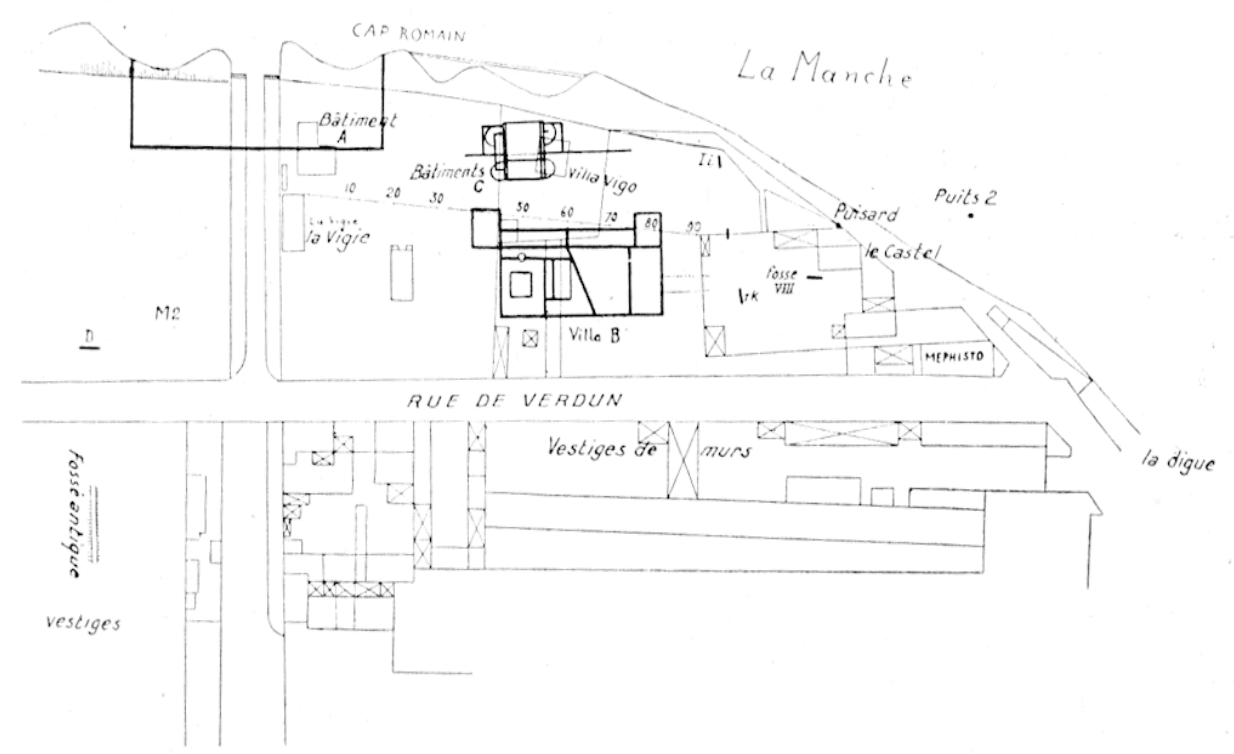

Pri. 1. - Plan géneral de silualion: les mines an Camp romain ì Sianl-ilubin.

(La presque lolalité des maisons est actueilenent rasée.)

également moins importante à mesure que l'on marehe plus à l'ourest vers Bernières où se retrouve le sable de la côte plate. Le terrain archéologique, qui porte naturellement le nom de C'amp Romain. ou de Castel, s'étend au moins jusqu'à la rue de Verdun qui longe la coole à environ $70 \mathrm{~m}$. Il ne dépasse guère ver's l'est l'emplarenient de l'hòtel du castel, brûlé à la Libération, el la digue de Saint-Aubin, Inndis qu'il peut se prolonger a l'ouest sur la commune de Bernières, la division administrative actuelle étant des plus ricentes (1852) el ne correspondant en rien à l'étal

(1) Bull. Soc. Ant. Norm., XLIX, 1942-19:45, p. $433 \mathrm{sq.}$

(2) Sur les origines du culte de cette Vierge Noire, of. maintenant une note de $M$. LAciotkt,e, ibid., L, 1946-48, p. 2.42 s(f., qui souligne langle prononec par la voie romaine

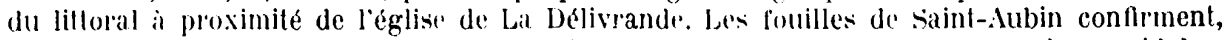
scmble-t-il, les vues de ce savanl sur l'attitude des cinceliens en face des anciennes idoles. 


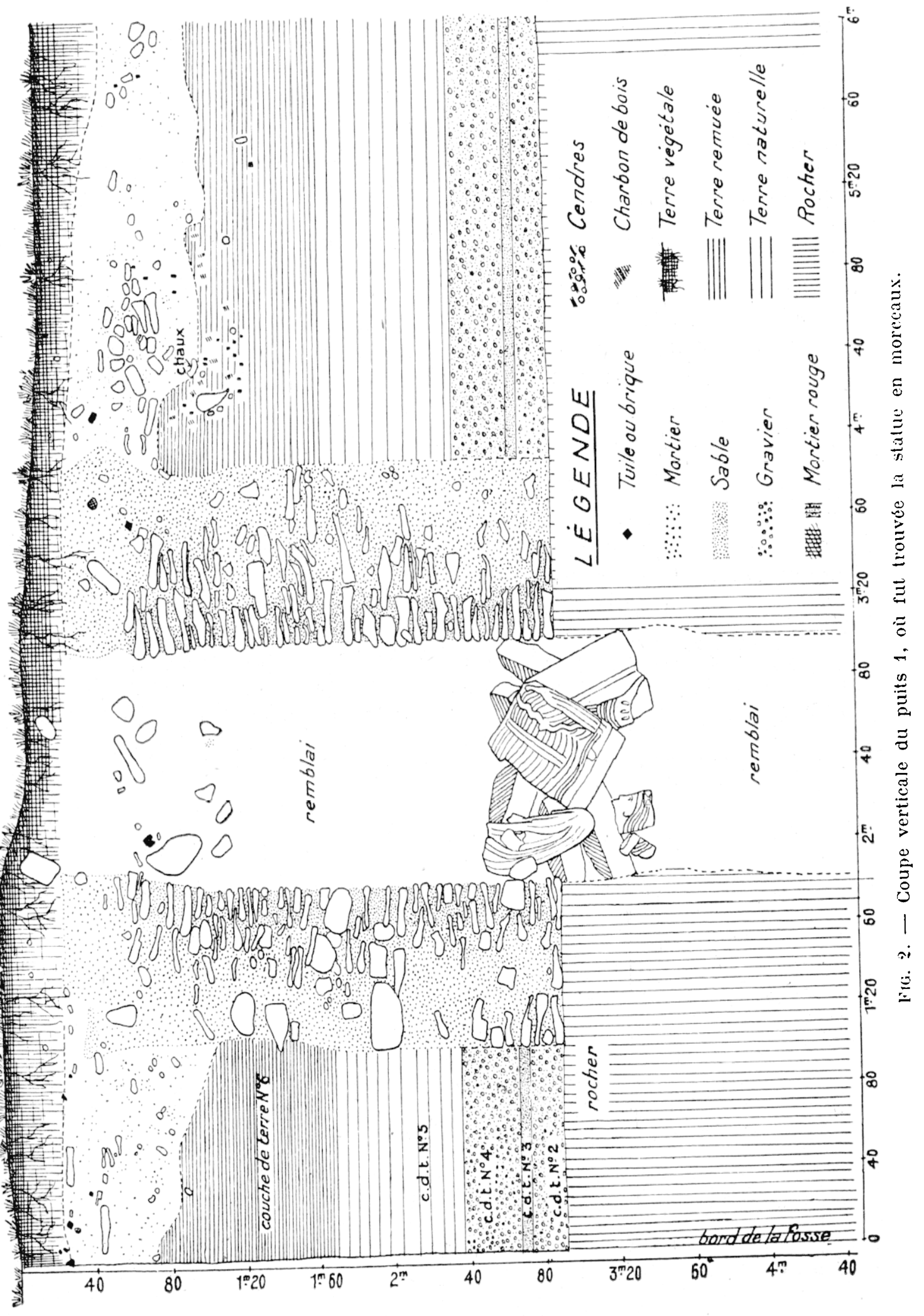


ancien du pays. Ln chemin de descente vers la mer entaille la falaise un pẹ à l'ouest du Cap Romain.

Le sol est facile à observer à la coupure de la falaise et dans divers puits (fig. 2). Sous une couche d'humus de 0 m. 35, où apparaissent fréquemment des fragments d'ossements et de poleries, on trouve sur $1 \mathrm{~m}$. 35 de la glaise rougeàtre mêlée de pierres gréseuses, de morceaux de tuiles, de charbons de bois et de débris de mortier à la chaux. Au-dessous, le sol vierge est formé de glaise jaunitre reposant sur un lit de graviers ferrugineux, un autre de sable blanc verdâtre et enfin sur la roche gréseuse qui, à la falaise, se désagrège facilement en pierres plates. Les couches de glaise correspondant géologiquement aux dernières glaciations et de grandes pierres visibles a la cote et jusqu'assez loin en mer sont des blocs erratiques ${ }^{1}$.

Depuis longtemps l'attention des arohiologues et des amateurs d'antiquités avait éte attirée sur le (amp Romain ou itaient fréquentes les déconvertes fortuites de sépultures, d'objets antiques et rn particulier de monnaies: l'affouillement de la frilaise parl la mer révéla d'ailleurs constamment les niveaux areheologigues. ln bon résume des observations ef recherches qui ont été failes sur le sitr jusqu'a la dernicre guerre a cité

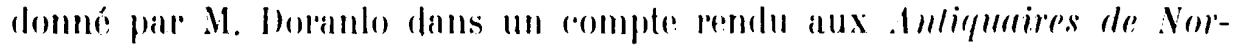
mandie ${ }^{2}$. J'en retiondrai les points suivants:

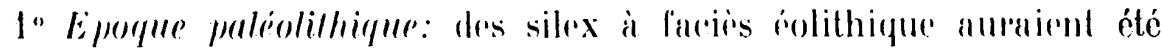
reereeillis par 1 . Ilue a la base de la falaise ". Le's bloes erratigues de la cote seraient, d'apres J. Tirard, des migalithes renversés ';

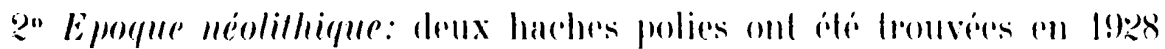
dans les ćboulis de la lalaise ";

3" Age de bronze: une hache plate i bords relevés all marteau. a été déeouverte an 1910 dans des travaux de terrassement pròs de la gare ${ }^{6}$;

4" Epoque historique: en 1835, on aurail dégagé. "ourant du nord au sud, "au Vieux Castel. un mur de plus d'un mètre d'épaisseur; long d'une dizaine de mètres. auquel s'en ratlachaient d'autres de moindre importance dont l'ensemble figurait de pertites cellules dont la destination

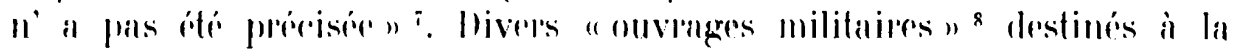

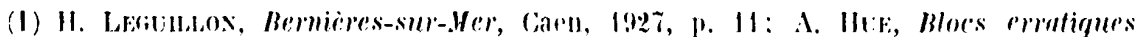
duns les enrirons de Luc.

(2) Bull. Soc. Antiquaires de Normandir, NLVIII, 1940, 11. 540-54.5.

(3) A. Ilote, ol). laudl.

(i) Bull. Soc. Ant. Norm., XVI, 1892, p. 188

(5) Ilide.

(6) Ibid.

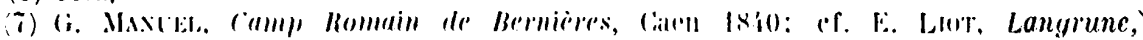
(aen, 1895, p. 48. pp. 10-19.

(8) Dr Doranlo, Bull. Soc. Ant. Norm., XXXV, 1924, p. 427; H. Legullon, op. laud., 
défense de la còte ou de l'entrée du petit port de Saint-Aubin auraient aussi existé et l'on rovait encore en 1894 les ruines d'une chapelle où l'on trouvait des cercueils et des squelettes" ". Plusieurs puits au pied de la falaise ou à quelque distance en mer étaient connus des pêcheurs, ainsi que des "aqueducs" lepérés en 1923 vers Bernières à l'occasion d'une marée exceptionnelle ${ }^{2}$. In peu partout, on signalait des tombes, sarcophages de pierre ${ }^{3}$ ou fosses dallées en pierres plates ${ }^{4}$, des tuiles romaines, des monnaies et jusqu'aux fragments d'une oll deux statues disparues aujourd'hui ${ }^{6}$, des tessons de sigillata ${ }^{7}$, des cuillers, des bracelets de bronze.

En résumé, il était évident qu'un habitat gallo-romain, succédant peut-être à une occupation plus ancienne, s'était installé sur le promontoire de Saint-Aubin. Mais le site. couvert dès le $\mathrm{xix}^{\mathrm{e}}$ siecle de villas et d'hôtels, n'avait jamais été l'objet de fouilles sı̀stématiques.

\section{Les fouilles de 19.92-1944.}

Arrivé à Saint-Aubin en mar's 1942. je trourai le terrain traversé par des tranchées (fig. 3). Trois blockhaus étaient déjà construits dont un au Cap Romain mêne. Des fosses avaient été creusées, notamment pour l'implantation d'un phare (1 9 du plan), sans qu'on se fint avisé que les murs antiques ou les tombeaux coupés par les travaux de défense pussent présenter un intérêt quelconque. Ce furent des fragments de sigillata qui attirèrent d'abord mon attention, mais pendant trois mois je ne pus faire autre chose que de collectionnel les trouvailles faites an hasard.

A partir de mai 19'2 je mis én place un système d'arpentage et je m'attachai à relever les diver's restes antiques qui apparaissaient au fond des tranchées ou des fosses d'abris bétonnés. Naturellement c'étaient seulement les nécessités militaires qui dictaient le plan des travaux et ce

(1) Bézières, Cf. PILlet, Mém. Soc. Ant. Norm., XII, 1840-41, p. 410.

(2) J. TIRARD, Bull. Soc, Ant. Vorm., XVI, 1892, p. 178.

(3) PILLet, l. l., p. 410.

(4) Drs Doha.io ef Gossista, Bull. soc. Anl. Norm. Xlvill, 1940, p. 514.

(5) Deux petits bronzes dont un Victorinus au Castel (Bull. Soc. Ant. Norm., IV, p. 379); sept medailles, de Claude, Trajan, Varc-ilurile et Constantin it la fouille dun grand mur au Cap Romain (E. Liot, Lantrune, Dl. 49-50); d'autres, de céron, et de Constantin à Bernières (Bull. Soc. Ant. Norm., XXXV, 1924, p. 420): une monnaie en electrum des Baiocasses, un aureus d'luguste au revers de Cä̈us of Lucius Caesar (ibid., I. IN60, p. 355 ), etc.

(6) Bull. Soc. Ant. Norm., I, 1860, pp. 291 et 369; XVI, 1892, pp. 150 et XXXII, p. 275. Il s'agit d'une stalue nue assise, en grès, de petite taille, et d'une jambe en trois fraginents.

(7) Notamment des signatures MVXTVLLVM, GE.VTILIS et un morceau de vase de Lezoux (Bull. Soc. Ant. Norm., XXXV, 1927, p. 420). 
n'est qu'exceptionnellement el au prix dr grandes diffieultés que je pus sur cerlains points pratiquer de rais sondages archéologiques ${ }^{1}$.

Les conditions dans lesquelles furent faites les fouilles, l'impossibilité où je me suis trouvé d'effectuer depuis lor's aucune vérification stir place clonneront forcément à ce rapport un caractere fragmentaire al provisoire. Bien que l'on ait louve asser souvent des outils ou des pointes de fleches en silex laille retument, des roduillages (moules de huîtres), des défense's de sanglier ol mêne un andouiller de cerf travaille, toule élude stratigraphique ful pratiquement impossible dans les plus anciens niveaux de civilisation: le sol avait été si remué pour les lavaux des champs a les lanchées des fondations postérieures que, la plupart du temps, les objols prehistorigues apparaissaient hors de tout contexte datable. d'ai semlement noti l'absence, asse\% singuliere sur l'ensemble du lerriloire foutli en 1942-4.t, de hacheltes de piere et de mobilier de l'àge du Bronze. Il n'est pas impossible qu'on en trouve un jour daus quelque dépot volif, ou les auraient grouprés la pición on la supersdilion des Gallo-Romains ${ }^{2}$.

C'est vers l'époque gallo-pomaine surloul, si riche en vesiges de forles sortes a saint-ilubin. que s'est portie mon atlention. Le dibut en

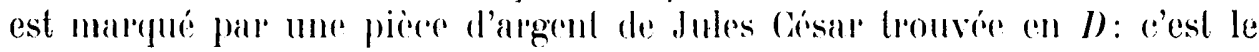
plus ancien document date de la région. On se rappelle les expéditions de I'. Crassus of Q. Tiburius Sabinus daus les provinces de l'Ouest en 57-56 av. J.-C. ${ }^{3}$ ef l'on imagine que les Romains aient pu s'intéresser asso\% vile a la coole de Basse-Normandie. ou Saint-Aubin esl fort bien

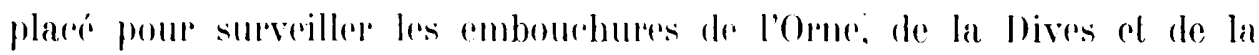

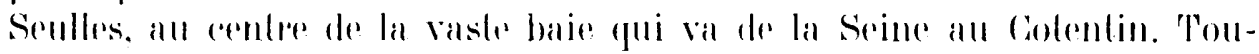

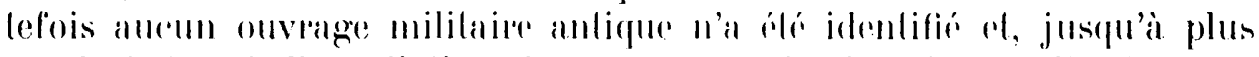

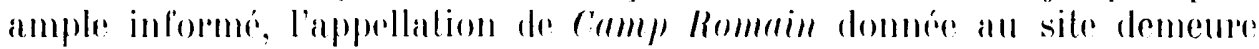
tout à lail graluile?

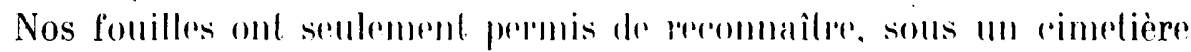

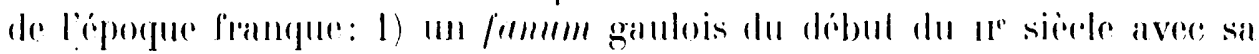

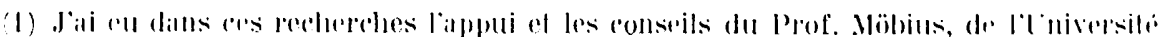

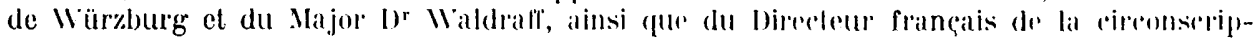

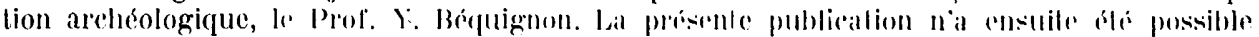

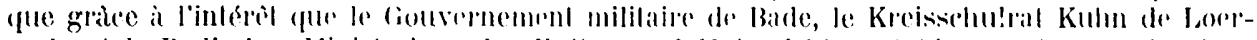

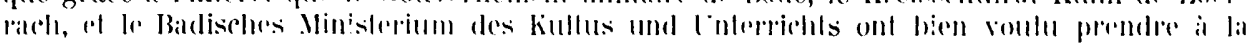

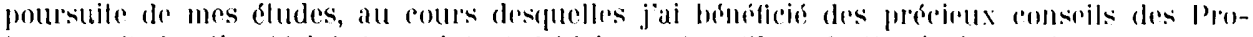

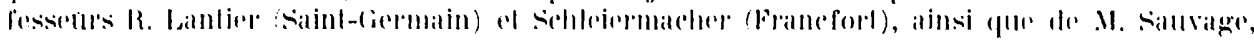

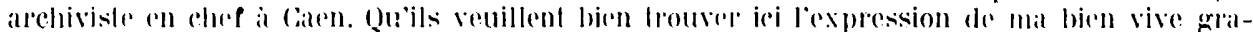

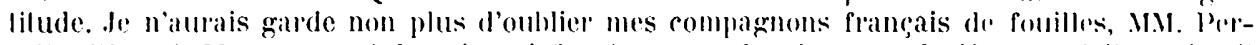
refle, Plumel, Mareheron ol Jamais qui firenl preuse du plus grand gèle quand il salgissial

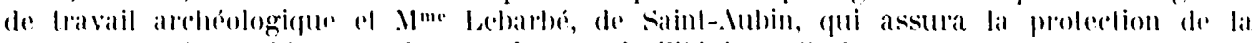
plupart des trourailles el mia grandement facilité leur élude.

(2) De lels dépòts de hachetles de pierre ont ale souvent tromvés piatl de lesily

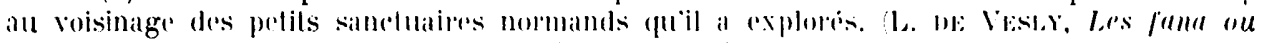
petils temples gallo-romains de la région mormande, linuen, 1909, pussim).

(3) Cars., B. G., II, 3' et III, 17 sq.

(i) Cf. Leglldon, Bernic̀res, pp. 18 et 76. 
slatue de rulle, ses annexes ol son péribole; - 2) quelques indices d'un sancluaire cellique plus ancien; - 3) une villa gallo-romaine lardive arant englobé le temple gaulois; - - 4) un bahnéaire sans doute destiné i la rilla; -5 ) plusieurs amores de constructions non digagies. Je vais passer en revur aes diverses découvertes en rappelant que la stalue ganloisere trouvée brision dans un puils près du famum, a déja ciló publiće par 1. Bérguignon, qui a domé à relle oceasion des indicalions précieuses, mais forrement loop rapides, sur l'ensemble dre la foutler.

\section{1) Le Frommm grumlois.}

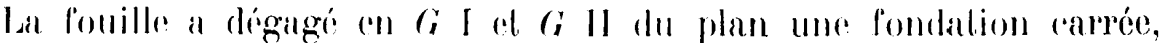
exactement orientio selon les quatre points cardinaux, de $11 \mathrm{~m}$. de côte

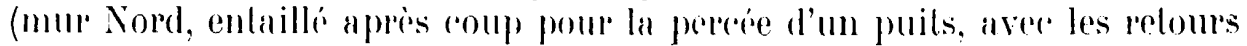
l'angles Est el Ouest ainsi que l'angle Sud-Ouest). Elle encadre une seconde fondation sans doule de mème forme el concentrique (mule Nord et retours d'angles seuls dégagis), mais de 5 m. de roli seulemenl. Ces

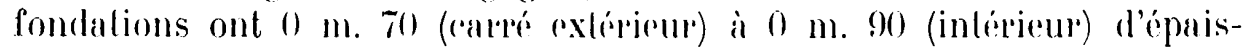
seur el sonl faites de grands blocs de gris bien assembles an mortiel de chaux. Les restes d'un dallage de petiles picres gresenses subsistent it l'inlirieur, entre les deux fondalions. comme dans la plus petite. Des dábris de luiles à rebord rouges el jaunes álaient entassés toul autour.

Il s'agil évidemment là d'um petil fromum de lype désormais classigue. dans le Nord de la Gaule, les Iles Britanniques, la Suisse el sur les bords du Rhin. En parliculier il est très analogue à ceux qui ont élé éludiés far de Vesly dans la région de la Basse-Seine ${ }^{2}$. La fondalion rentrale plus solide ciluil celle du sancluaire proprement dit; la fondation extériente ne supportail qu'une serpe de piliers (de bois?) formanl priau ou galerie, avee rouverdure do luiles. La dale n'ese fommie jusgu'à présent

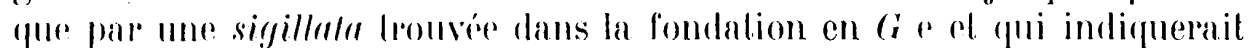
le II $^{*}$ siecle ${ }^{3}$. In pelil fragment de marbse provenant du même endroil apparlenait peut-ôtre au revêtement des murs.

Vers l'Est, une fondalion plus légère divisée en deux eomparliments éroits par un mur de refend Nord-Sud, avec un dallage en pierre de

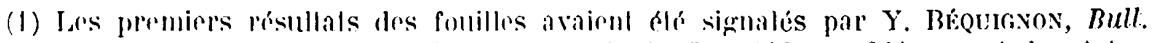

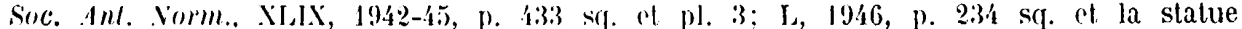
est. publiée par lui dans les Monuments P'iol, XIIII, VI, p. 833 sq. et pl. XI, ou l'on trouvera les références aux autres reproductions et études. On notera que ce savant parait hésiter sur la date de la statue: III $\mathrm{s}$. dans l'article du Bulletin, 1946, p. 239 et $\mathrm{I}^{\mathrm{er}} \mathrm{s}$. dans celui drs . Vonuments riol, p. 91. La deseription qu'il fait d'autre part du fammm graulois, (ibidl., p. 94) n'est pas absolument. satisfaisante, car M. Béquignon n’a pu disposer pour ces publications provisoires que de documents et renseignements incomplets.

(\%) DE VESLY, op. laud., p. 115 sq. Cf. la carte de la planche VIII dol colfe qui a éte donnee par Whrester, Antiq. Journal, VIII, 1928, pl. XINII. On pourra rneore ajouler le fanum de Saint-Aubin du Vieil Evreux, Gallia, II, 1943, p. 191, sq.

(3) Ene monnaie de Mare Aurble a sté trouvée à ce niveau en ./ 3 . Detix monnaies

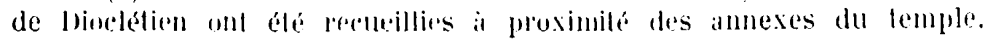


grès, doil correspondre à une amnexe quelconque. Elle ílait garnie d'un revêtement d'enduit rouge pompéien dont un fragment a ćlé recueilli. Aucune trouraille n'est encore venue nous fixer la destinalion de ces deux pièces interprélées ailleur's par de l'esly comme la loge du gardien et le dépôt ou le magasin de vente des offrandes ${ }^{1}$.

Au Nord, à une distance de $21 \mathrm{~m}$., un long mur Ouest-Est est interrompu dans l'axe du temple pour former une sorle de salle de même orientation que le temple, mais sans murs transversaux identifiables: il faut croire qu'une cloison légère fermait pourtant la salle puisque des conduits d'hypocauste semblent aroir existé dans les fondations des murs latéraux". Lne pièce de Maximien coupée en deux el une autre percíe d'un trou (amuletle) ont élé trouvées au roisinage de rette salle. C'est sans doute aussi de là que proviennent les cuiller's de bronze et les bracelets recueillis nutrefois sur le sile ". Le grand mur ne peut être que le péribole du temple, avec une entrie ou salle de réunion.

Les meilleures comparaisons a proposer pour cel ensemble nous parraissent être les famu de la Mare du l'uits et d'Orgeville qui présentent le même dispositif d'enceinte arec: salle (dre réunion?) et mieux encore celui d'Harfleur dont l'orientation spéreiale répond aux mêmes nécessités qu'à Suint-Aubin (fig. f) ${ }^{4}$. Normalement les lemples sont ouverts a l'Fst:

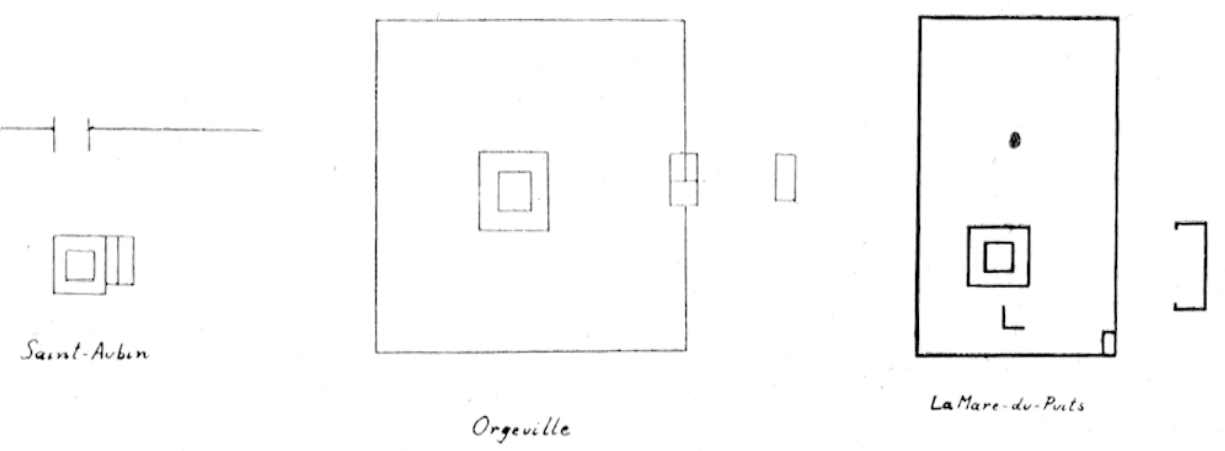

Fic. 4. -- Comparaison entre to famum de Sainl-.lubin el ceux de La Mare-du-Puits et dorgeville.

à Harfleur, pour faire face à la Seine sans doule, le lemple donne au Sud et c'est l'annexe qui est adjacente au côté oriental. A Saint-Aubin, l'annexe est placée de même ê le lemple devait être ouvert au Nord pour dominer la mer. Tout vestige de perron ou d'entrie a disparu cependant quand le puits a ćté creuscí au milieu du mur Nord.

(1) DE VISLY, op. laud., p. 126.

(2) Le meme dispositif est signale a Orgeville, ibirl. p. 131. C. allssi les enceintes

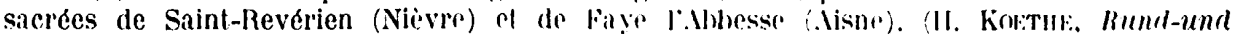
l'lerechtempel der Kaiserzeit, 23. Bericht der Köm. (ierm. Kommiss. 19333).

(3) Des découvertes similaires sont mentionnées a la rité de limes, au catelier de Criquebeuf, etc. De VesLY, op. laud., pp. 12, 51 et 114-145.

(1) DE VesLy, op. laud., pp. 19, 22 et $121 \mathrm{sq.}$ 
Sud

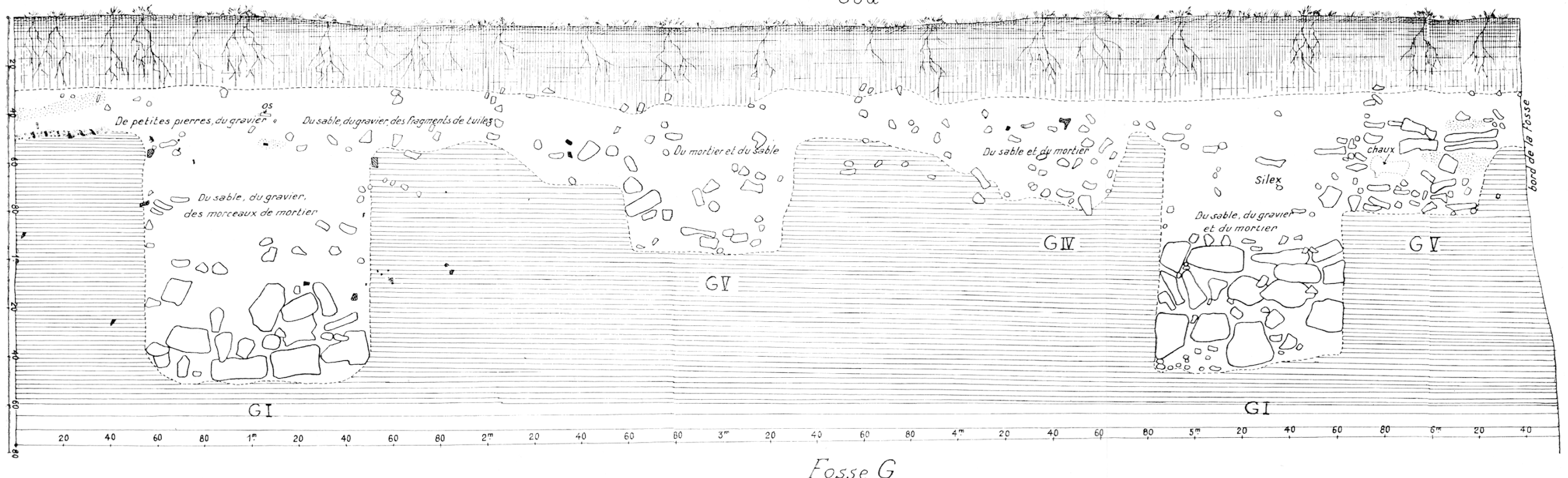

\section{Ouest}
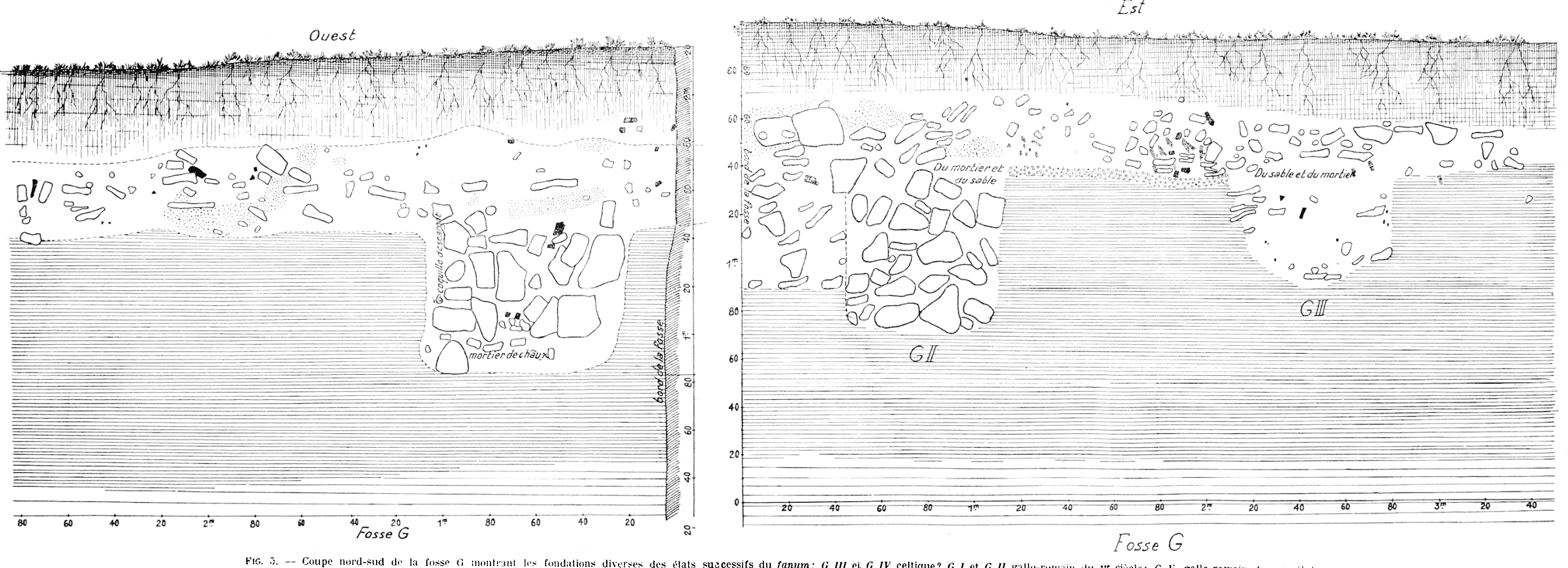

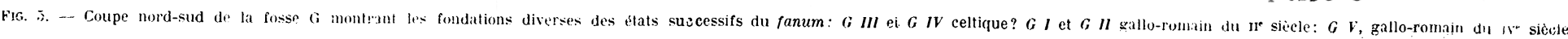


C'est dans ce puits qu'à $2 \mathrm{~m} .50-3 \mathrm{~m} .40$ de profondeur (fig. 2) ont été trouvés les cinq morceaux de la statue de déesse assise, encadríe de deux enfants, qui est maintenant au Musée des Antiquaires de Caen. Des éléments du socle avaient élé igalement découverts dans le puits. Ils ont malheureusement disparu dans des travaux d'empierrement au voisinage. C'est la première fois qu'une statue de culte peul être mise en toute certitude en rapport avee un tel fanum. L. de Vesly n'arait dérouvert dans les cellix que des fondations de piédeslaux, ou parfois certains aménagements plus complexes, comme ì Orival ${ }^{1}$, qui lui faisaient supposer l'existence d'une chambre oraculaire. On serait tenté de mettre l'évidement qui existe ì la face postérieure du siège de la diesse en rapport avec quelque dispositif analogue ${ }^{2}$.

Pour en terminer avec les annexes du temple, signalons au roin S.-(). de la fosse $I$, en dehor's du péribole, mais à peu de distance, une excavation corrée de $0 \mathrm{~m}$. 60 de profondeur $(2 \mathrm{~m}$. $\times 2 \mathrm{~m}$.), à environ $1 \mathrm{~m}$. 20) du sol actuel: le fond et les parois itaient garnis de tuiles juxtaposées et la fosse était pleine de débris de chaux el de mortier rouge. On aimerait considérep cette fosse romme une piscine (cultuelle? celtique?), plus tard utilisíe, ainsi qu'une autre en $B$ f, comme fosse à chaux. Ies bassins analogues sont signalés par de Vesly à la Mare du Puits et all famum de Criquebouf ${ }^{3}$.

\section{2) Le Sanchuaire celtique (?).}

En $G$ III et $G$ IV du plan, la fouille a reconnu les truces d'une tranrhée de fondations, de direction Est-Ouest, avec un retour à l'angle N.-O. Dans son état actuel, comme le montre la grande coupe do la fosse $G$ (fig. 5), la tranchée élait simplement remplie de terre, de sable, de mortier, de quelques blocs de grès et de petits morceaux de luiles, mais ce remblai était facile à distinguer de la contexture nornale de l'argile vierge. Au bord de la tranchie vers le Nord, des pierres de fondations arrachées el une grande quantité de charbons de bois étaient encore visibles sous le dallage ancien du temple gallo-romain, tandis qu'au sud du fossé une couche de graviers fins el de sable de $0 \mathrm{~m}$. 04 d'ípaisseur gardait la trace de charbons de bois de mêmes dimensions, réguliorement espaces en largeur de $0 \mathrm{~m}$. 03 .

II y avait lì de toute évidence une construction antéricure au famum gallo-pomain, aver des murs tout ou portie en bois sur un socle de

(1) Ilbill., p. 82.

(2) M. Béquignon n'a pas mentionné cette particularité du siège qui n'est visible sur aucune des pholographies qu'il présente.

(3) DE VESLY, op. laud., p. 104. 
pierres el une grille de bois ou claie (en guise de porle?) ${ }^{1}$. Nous n'avons aucune indication sur la destination de l'édifice, dont les fondations furent arrachees el le sol de graviers mulatle lors de l'implantation du

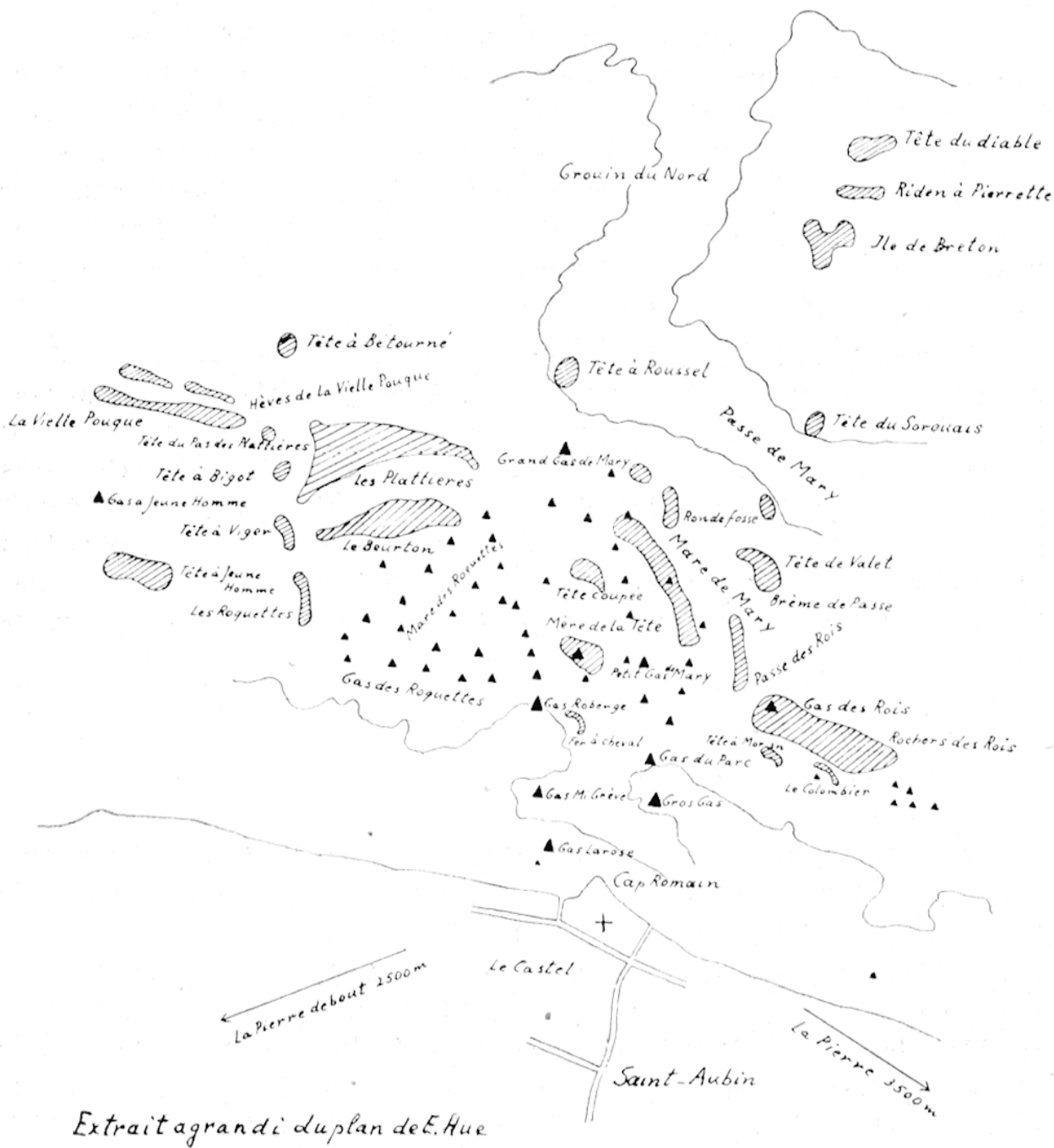

- Menhir socher sous-marin $1: 20.000$

$\triangle$ La Haute Devise

Fro. 6. - Jos "blocs erratiques" drvant la cole de siant-lubin.

(1) Pour de semblables ronstruetions de bois, of. II. Iöscinkl:, Triere. Zeilschrif!. V, 1930-31 et. H. Koftue, op. laud. Au sud du lemple de l'allhachlal, H. löschke trouva

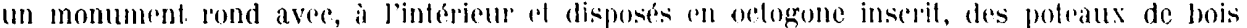
encastrés dans des pierres de taille. Une grille cxistait entre les poteaux (Dic Erfor-

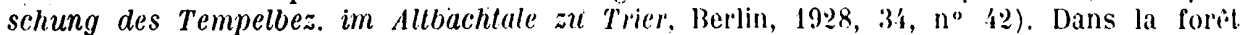
de Coblener, un ancien temple carré en billes de bois avial été frécédé d'une construrtiun en bois sur léger soubassement de piorre. 
sanctuaire gallo-romain. Mais la persistance très générale des lieux de culte aux mêmes endroits ef l'obserration des prétendus blocs erratiques de la côte nous font penser qu'il s'agil peut-être déjà d'un bâtiment religieux f'ort ancien. En effel, un simple coup d'œil sur' les relevés (fig.6), qu'E. Hue a donnés de ces "Gas ", auxquels les pêcheurs ont attribué des noms piltoresques ${ }^{1}$, laisse apercevoir comme une allée menant du Nord vers l'emplacement du temple. Toutefois avant de développer un parallèle avec Stonehenge ou les alignements bretons el avant d'affirmer l'existence d'un sanctuaire cellique, antérieur au famm gallo-romain, sur un emplacement déjà sacré à l'époque mégalithique, il conviendrait d'attendre et le dégagement complet de la rígion du temple et la mesure précise des positions des "Gas".

\section{3) Ia villa gallo-romaine.}

Le fanum gaulois était-il isolé? Appartenait-il à un ensemble de lieux de culte comme il s'en est déjà rencontré en Normandie où les petits temples sont parfois très rapprochís les uns des aulres? Est-il en relations avec un habilal, quelque hamean de pêcheurs ou quelque station de soldats? Nous serions bien en peine de le dire et nous pouvons seulement rappeler les trouvailles de fragments de statues à Bernières et ì Tailleville, tout à côté de Saint-Aubin et la découverte, hors du Camp Romain de Saint-Aubin, au sud de la rue de Verdun, de fosses et de murs antiques qui n'ont ju ètre convenablement ítudiés.

En lous cas, les construclions dégagies au voisinage du sanctuaire ou sur son emplacement appartiennent à une période bien postérieurs et ne sauraient à notre sens remonter plus haut que la fin du $1 \mathrm{II}^{\mathrm{e}}$ siècle. Elles forment une villa rustica aver deux lours relices par une galerio et une grande salle en arriere ${ }^{2}$. J’y ai distingué plusieurs états de cons. truction.

a) Premier étal. A l'est du lemple, s'ílevèrent une lour de $6 \mathrm{~m} .40 \mathrm{sur}$ $8 \mathrm{~m}$. 20 et un grand bâliment, divisó en deux salles $I$ et $E$, qui s'appuỵail au mur Est de la galerie du temple, renforón et surólevé. Les autres élíments du famm. cella, galerie nord, ouest (el sud?), annexes devaient être en ruines el ne furent pas relevés. On se contenta de creuser un puits en entamant l'anciemne fondation nord de la galerie et de bâtir i côté, avec les pierres de la ruine, une petile chapelle $G \mathrm{I}$, sans doule pour

(1) Cf. J. Lecoevr, Moniteur du Calcados, 11 juin 1880. Le nom de Vieille Pouque est en particulier très caractéristique et rappelle celui de Pouquelér, pierre fée, donné volontier's à des menhirs. Certaines pierre's levées subsistenl ou subsistaienl rncore naguère sur la falaise. C'est ainsi qu'on mentionne près du Castel la Haute Devise qui peut être simplement un milliaire romain, et la Pierre Debout, vers Bernières.

(2) Ce type est des mieux connus, ef. A. (Bkerieh, Manuel, VI, 2, 2, p. $793 \mathrm{sq}$. el nolammenl fig. 277; la villa de Lebisey est peu éloignée de Saint-Aubin. 
abriter la statue de la déesse. dont le culte restait encore vivace ${ }^{1}$. C'est de cetle époque que dalent le chemin pavé qui joint le puits à la villa et sans doute aussi deux autels qui ont été découverts, l'un au Nord entre le lemple el la salle du péribole, maintenant transformée en balnéaire, l'autre dans un four de ce balnéaire même.

Les fondations qui seules subsistent pour le premier élat de la villa (fig. 7) sont d'une construclion très solide et soignée: deux rangées de dalles de grès, la seconde arec ses blocs placés de champ, et une couch"s de $0 \mathrm{~m}$. 30 environ de pierres plates noyées dans du mortier; le lit d'attente dú mur élait très exaclement nivelé en ciment à la chaux. Les attaches des angles de fondalions notamment aux points 1 et 2 , à l'angle N.-O. de la lour (', et à l'angle extérieur $D$ b de la salle $D$ (fig. 8), étaient bien joinlovées avec calage des blocs par de pelits galetons. Au niveau de la lête de fondation, un dallage de gres subsiste dans la tour $B$, tandis qu'en $D$ c'est un parement en pierres de gri's et tuiles lices au mortier de chaiux. En $E$, le dallage a ćlé détruit s'il a jamais existé.

L'élévation de la lour a disparu. Elle devail faire une part importante a la construction en bois, car l'incendie du bàtiment a laissé d'épaisses eouches de charbons de bois all-dessus de la fondation. On a recueilli là, romme en $I$ ), de nombreux tessons de sigillata, des débris de verres et da fer's en abondance; il devait s'agir de pièces d'habitation tandis que $E$ pouvail n'être qu'une cour, une élable ou des communs. Comme la fouille a cle loul autre qu'exhaustive, il n'est pas possible de dire s'il n'y avail pas d'aulre's subdivisions de res pièces, ni quel était le mode de rouverlure ". C'est toul jusle si l'on a pu reconnaître le plan général. Les monnaies of les lossons de sigillutu domnenl une date assez nette à cet chsemble qui doil correspondre au l'enowreau ganlois après les invasions ('l les désastres de $275^{3}$.

Le puits 1 a un diamètre de $1 \mathrm{~m}$. 20 (fig. 2). La mangelle est faite de grands blocs de gries. Murdessous, sur $2 \mathrm{~m}$. 90, le puits est maçonné en pierres plales el morticr. Au-rlessous encore, et jusqu'à $8 \mathrm{~m}$. de profondeur où les infillrations d'eau arrêtèrent la fouille, le puits est taillé à vif dans le rocher. On y rerneillit, outre les fragments de la statue, charbons de bois, bloes de grès taillés, tuiles à rebord, tessons de vases galloromains, clous en fer, dents de porc, le cràne d'un chien et les squelettes de petits rongeurs. La construction du puits et son comblement sont aisément datés par les observalions suivantes qui permettent de résoudre le problème chronologique posé par M. Béquignon à son endroit ${ }^{4}$ :

(1) De tels lieux de culle annexés à une rilla sont bien connus: cf. Bonner Jahrb., 1333 (198), pp. 5L-59, pl. I-XIII; R.t..., 1930, p. 25:) sq.: А. Grexier, Manuel, VI, 2, 2, p. $793 \mathrm{~s}(\mathrm{q}$.

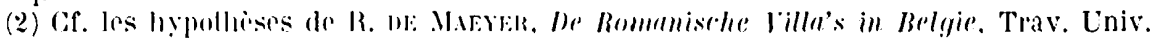
Gimd, 82e fasce, 193i.

(3) Ge. en particulier une monnaie do Licinius trouvéce en $M$ la dans la pièce $E$.

(4) Von. Piol, XIIII, p. 96. 
DECOUVERTES. A SAINT-AUBIN-SUR-MER
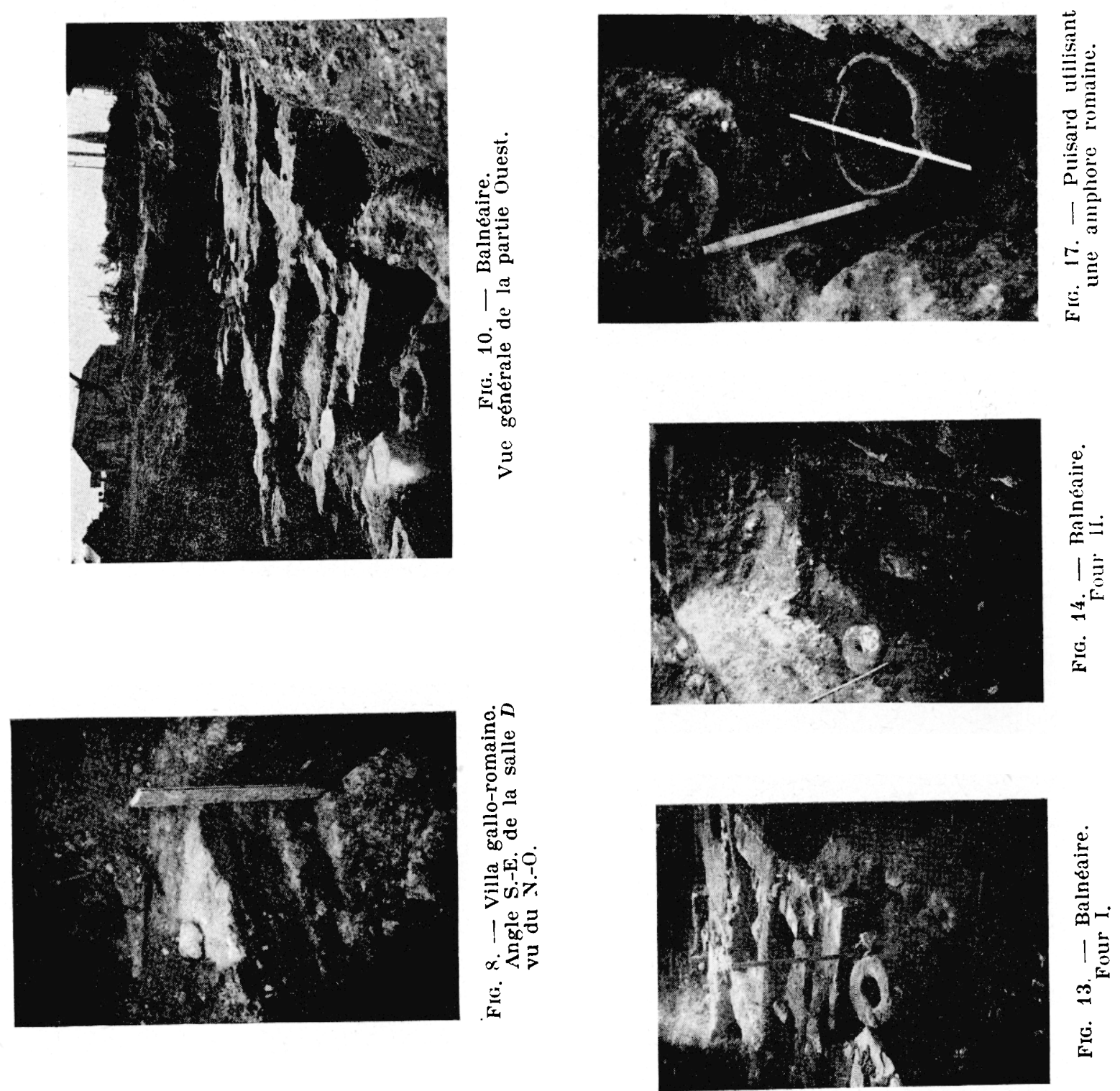

点
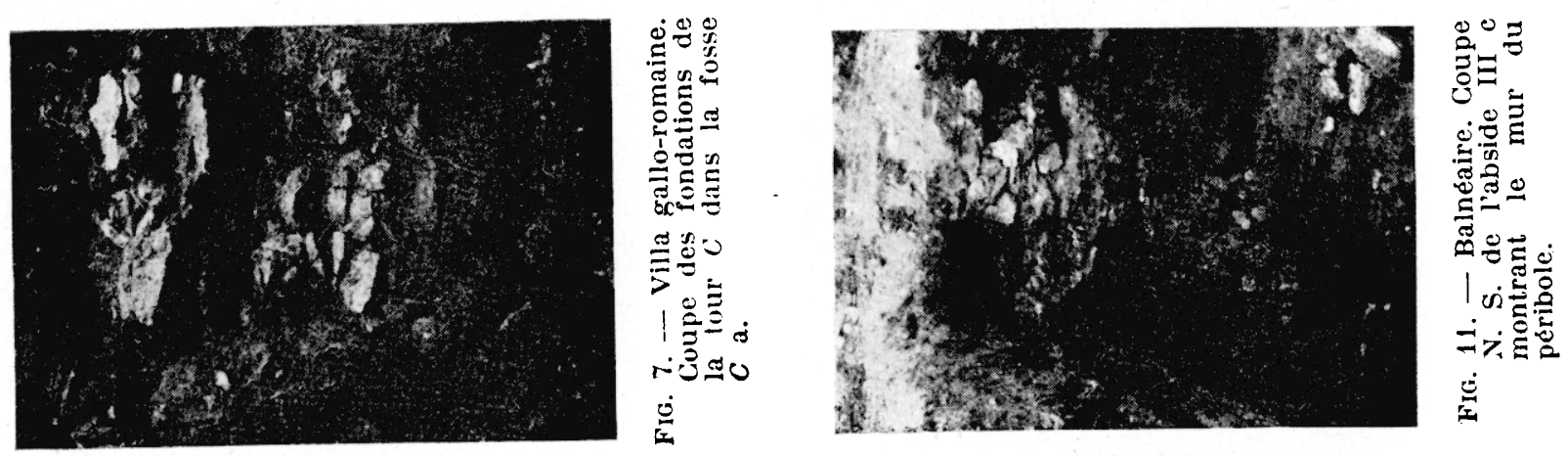
a) le puits est postérieur au fanum gallo-romain puisqu'il a fallu arracher une partie de la fondation de la galerie du fanum pour lui faire place; b) le puils est contemporain du premier état de la villa puisqu'il y est rattaché par un chemin dallé (pavés, tuiles, graviers, coquilles de moules pilées); c) le puits est contemporain de la chapelle $G V$, puisque la statue dut être abritie quelque part entre la destruction du fanum et la mulilation de la stalue suivie de sa chute dans le puits; d) le puits est antérieur à la pièce $G$ dont le mur $A c / B$ l repose sur la margelle; il fut donc abandomé lors de la réfection de la villa ( $2^{e}$ état).

Le puits et la ehapelle GV seraient done datables approximativement du début du ŕve sière el contemporains du premier état de la villa et du balniaire auxquels correspondent les autels romains et la demi-pièce de Maximien trouvée dans le balnćaire.

b) Deuxième élut. Vers la fin du Ive siècle, d'après les monnaies de Constantin el de Valère trouvées en $M I$ et $B I^{1}$, la villa fut achevée dans In forme classique des fermes à deux tours symétriques reliées par une galerie-façade. Pour cela, on construisit une tour $A$ de mêmes dimensions que $B$ el dallée de même. Toutefois les fondations en sont plus négligées. On prolongea ver's l'ouest le bâtiment $E$ en utilisant les fondations sud of ouest de l'ancienne galerie du temple pour former une pièce complète $G$. Ln indice caractéristique de cetle réfection est fourni par le petit trongon du mur Bo élabli pour relier l'angle sud-est de la tour $A$ à l'anrien mur onest de la galerie. Enfin. on réunit les deux tours et le bâtiment GED par deux fondations álroiles $B a, B d$ el $A(B) B l$ de 0$) \mathrm{m} .70$ et $0 \mathrm{~m} .50$ de largeur destinés à supporler soulement les colonnes du péristyle. Cette galerie couverte de $5 \mathrm{~m}$. 20 sur $31 \mathrm{~m}$. I0 ilait parée sommairement (pierres, luiles, graviers et sable).

Il est rraisemblable que les occupants de la villa étaient désormais des chrétiens: la chapelle fut détruite et la statue, brisée, jetée tête la première dans le vieux puits, aussitôt comblé pour faire passer le mur de fond du péristyle. Succédant à des siècles de consécration religieuse, la nouvelle pièce $G$ devint une cuisine, comme l'attestent les débris de vaisselle, les ípaisseurs de charbons de bois, de coquilles et de résidus d'alimentation qui furent trouvés au vosinage.

c) Troisième état. A une époque impossible à délerminer, un large mur oblique $S$ (fig. 9) et un pelit mur $B$ h vinrent diviser en deux la salle $E$ et le príristyle. Ce sont des fondations très négligées, débris de toutes sortes, y' compris des coquilles d'huîtres noyés dans un mauvais morlier, ou mème galetons sans mortier. La poterie trouvée à l'ouest de

(1) Cerst-it-dire dans le péristyle de la rilla. D'autres monnaies des mêmes empereurs ont éle trouvées notamment dans li balnéaire et dans la grande fosse $I$ creusée ¿ loulest entre le balnéaire et le batiment $A$, a proximité de la rilla. 


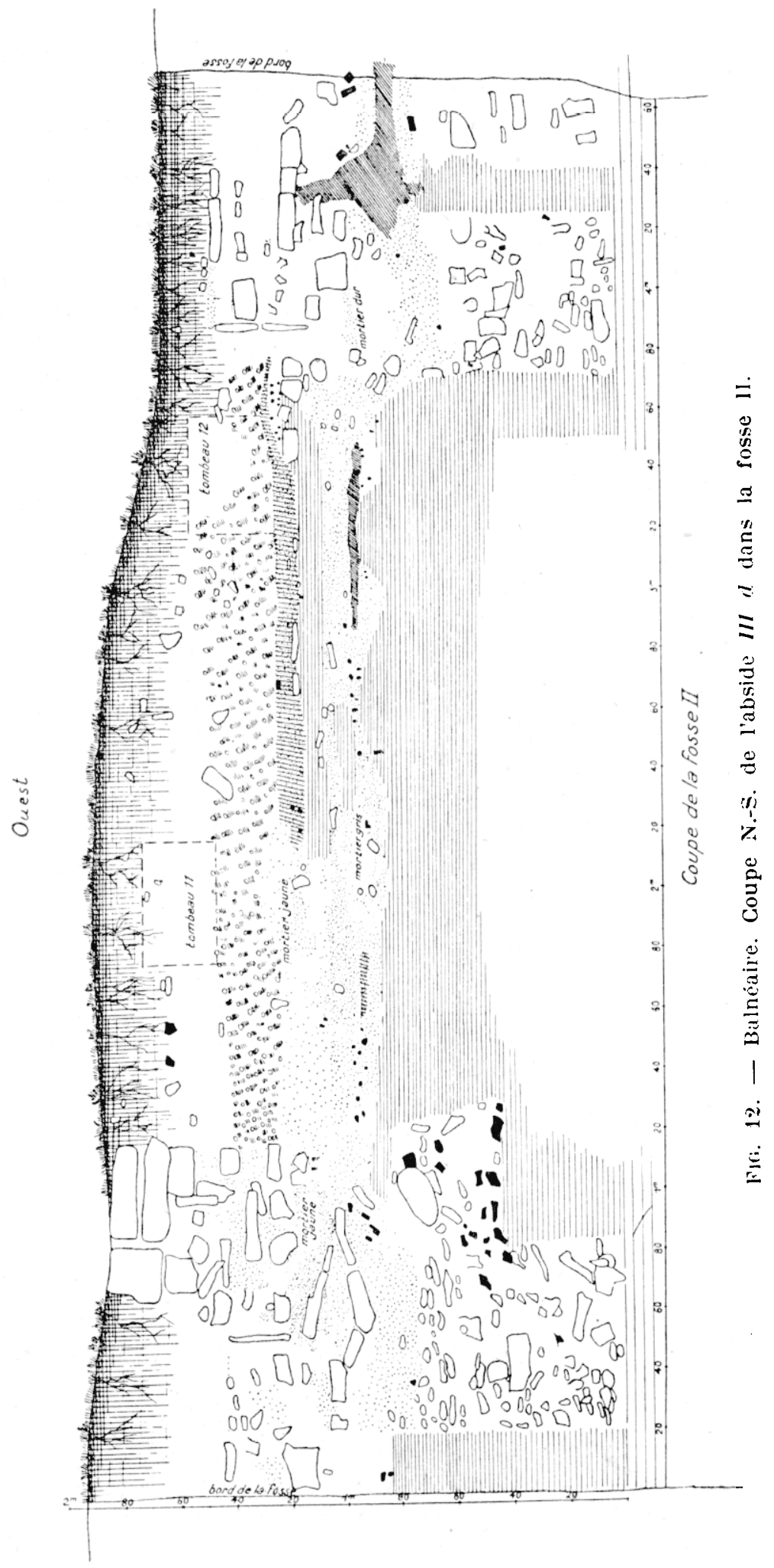


celle séparation paraît plus grossière que celle des pièces de l'Est oi vivaient peut-être les maîtres et leur famille ${ }^{1}$.

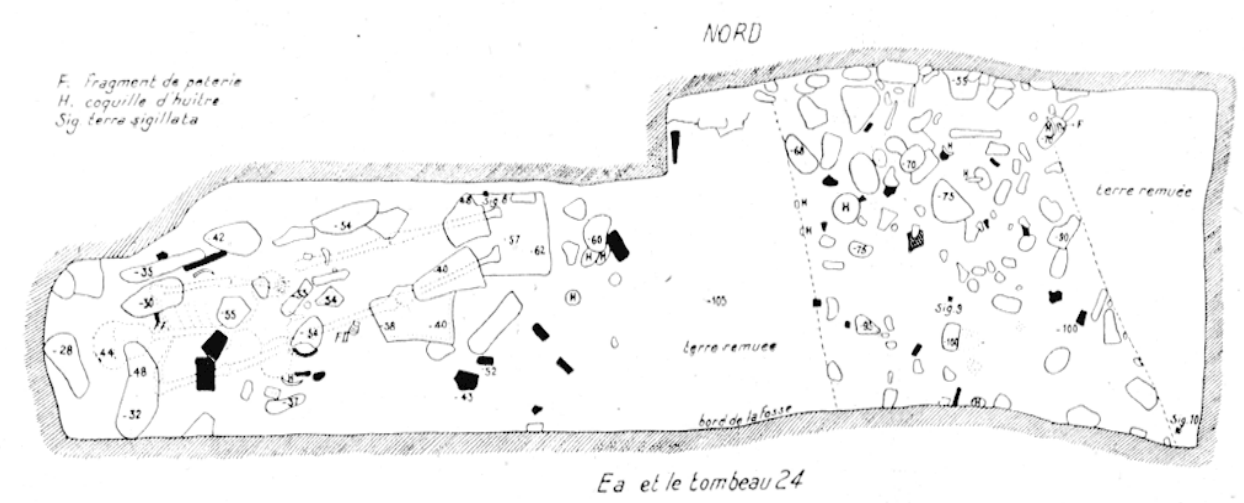

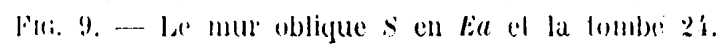

\section{4) Le balméaire.}

Il occupe l'emplacement de l'ancienne salle du péribole Nord (fig. 10). bes fondations soignées II élargissaient légèrement la pièce en lui donnant une dimension de $11 \mathrm{~m} . \times 11 \mathrm{~m}$. Cetle fois l'élévation des murs peul être observíe en quelques points: ils étaient en pierres de taille, couverts d'un enduil, et le dallage élait en mosaïque dont on a retrouvé un fragment. Deux annexes rectangulaires à l'Est (incompletement fouillées) et a l'Ouest contenaient les foumeanx. On y recueillil beaucoup de charbons de bois et de débris de poteries. I'n che'min dallé reliait au Sul le bahneaire an puils qui l'alimentait sans doule en eau.

Une réfection, probablement contemporaine du deuxième dat de la villa, vint ajouler a cel ensemble deux absides III e et III d. Leurs murs viennenl seulement buter sur ceux de la pièce II. mais ils sont solidement fondés $(1 \mathrm{~m}$. 06). L'élévation qui subsiste par endroits, sur une largeur de $0 \mathrm{~m} .50$ en double retrait sur la fonclation $(0 \mathrm{~m}$. 60 de large) est maconnée au mortier de chaux et porte un enduit à la face interne. In pavement épais de $0 \mathrm{~m}$. 40 (tessons de tuiles noyés dans un mortier, sur une rouche d'argile et un lil de chanx et de sable s'etend sur toute la surface des absides, recouvrant même en $11 \mathrm{k}$ le mur de l'ancien hypocauste (fig. 11 et 12 ).

Au nord de chacune des absides, une fondation rectangulaire abrite les fourneaux, qui sont logés entre l'épaisseur des murs II et deux démenls coubbes élroitement joints aux fondations rectangulaires. Len-

(1) Cf. La rilla romaine de la Forêt de Brotonne fouillée par Fatcuc et l'explication qu'il propose pour un mur oblique analogue (IIEm. Soc. Ant. Norm., X, 1836, pp. 367, 390 et pl. V, n. 2 et VI, n. 3-4). 
semble est de qualité médiocre, mauvais mortier, pierres disposées sans soin. Le fourneau $I$ a seul été fouillé intégralement. II comporte un dallage en pierres plates, un conduit de chauffe couvert de briques carrées plates, un foyer périphérique fait d'une meule près de laquelle était encore un tuyau de chauffage en terre cuite (fig. 13). L'autre foyer était fait d'une meule, d'un fragment de colonne et d'un fragment d'autel romain entourés de tuyaux de chauffage (fig. 14 et 15). Des écuelles, des

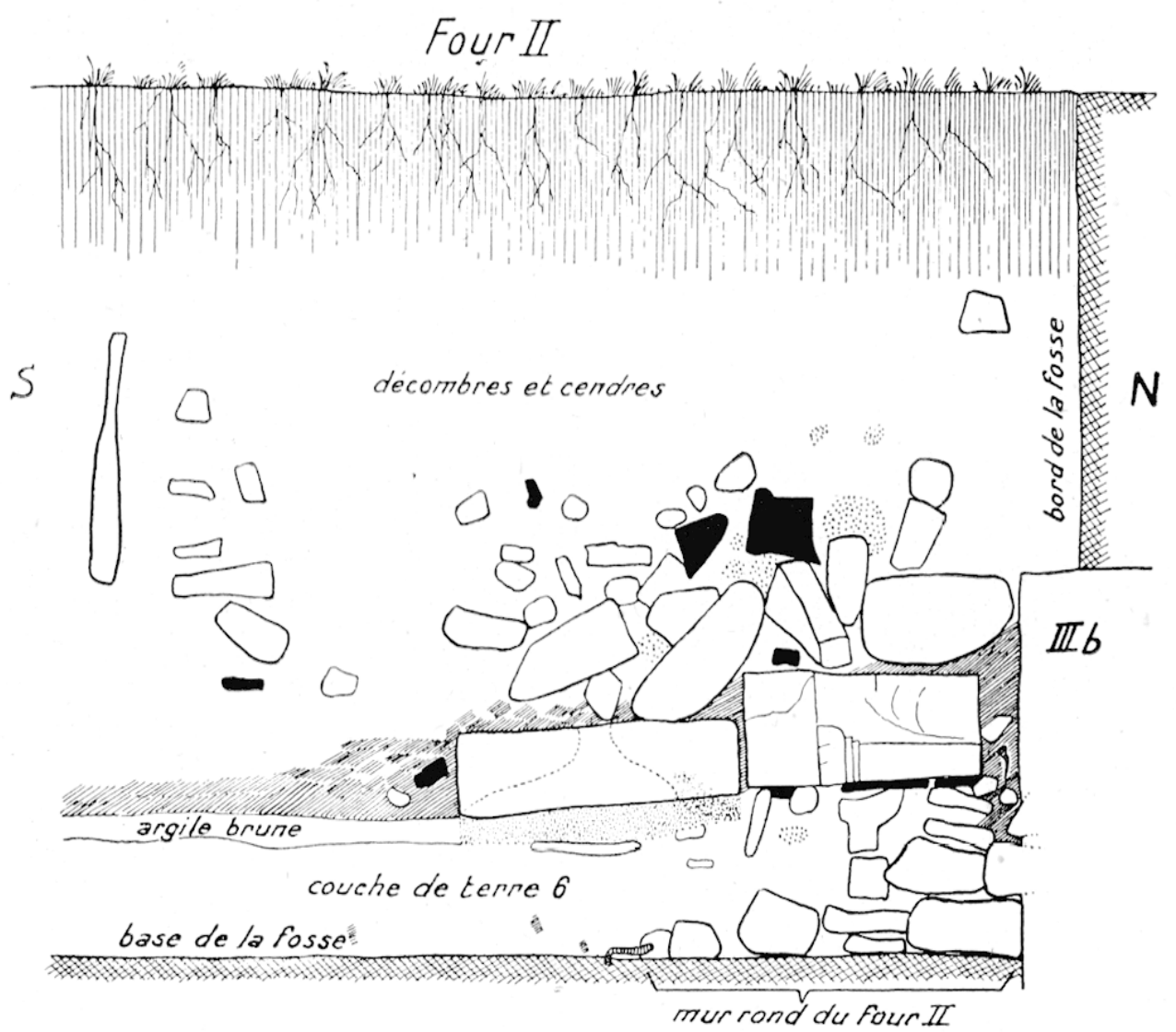

Fic. 15. - Balnéare. Coupe au niveau du four II.

vases à lait, beaucoup de tessons ont été trouvés aux environs, 'ce qui semble établir que les fourneaux servaient non seulement au chauffage du balnéaire, mais aussi à la cuisson des aliments. Au contraire, les fragments d'amphores (ayant servi à porter l'eau ?) sont nombreux dans les absides $I I I$ c et $I I I$ d qui devaient constiluer les étuves.

On se demande seulement d'où venait l'eau quand le puits 1 f'ut 'omblé. Les autres puits repérés, 2 et 3 , sont beaucoup trop loin. Je signale qu'une couche de mortier rougeâtre (chemin?) menait du balnéaire vers un anas de grandes pierres de grès à faible distance au sud, à la fosse II c. Etait-ce l'emplacement d'un autre puits? L'interruption des travaux n'a pas permis de le vérifier. 


\section{5) Amorces de constructions.}

Les temples sucressifs, la villa et le bahnéaire, s'ils constituent l'essentiel de nios découvertes, ne sont pas les seules constructions que nous ayons pu relever sur l'emplacement du C'amp Romain. Diverses amorces de chemin, notamment à l'est de $I$ ) el au sud de $E$ semblaient d'ailleurs souligner les liaisons de la rilla avee un ensemble plus vaste dont nous n'avons qu'une idée très grossière.

En $A$ el $D$ de grands buitiments onl idi identifiés vers l'ouest: leurs fondations ressemblent a relles de la villa $B$. mais leur élévalion devait contenir de trés importantes quantites de bois, car les tranchées ont révélé des couches épaisses de char-

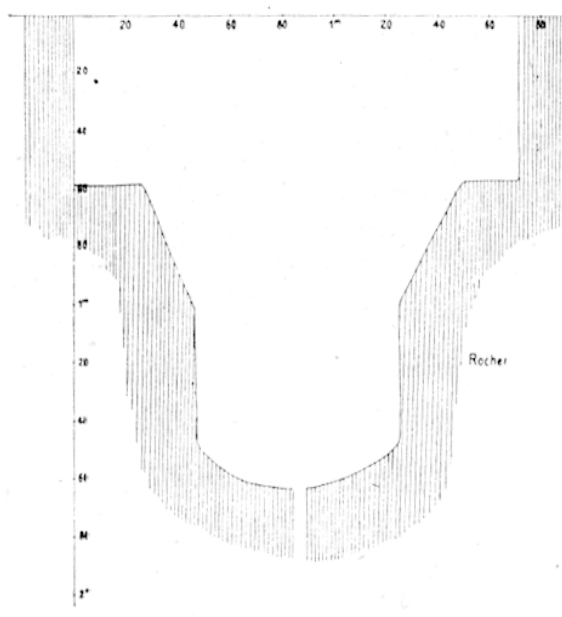
bons de bois. Plus au nord, d'autres ruines ont dû s'effondrer dans la mer qui ronge sans cesse la falaise: c'est là que passait jadis l'ancien "Grand chemin du Roi ", dont la plus grande partie a été arrachée aujourl'hui. Diverses fondations sont encore visibles aujourd'hui à marée basse. J'ai pu en particulier vider à environ $12 \mathrm{~m} .70 \mathrm{en}$ mer le puits 2. C'est un eylindre creusé dans le rocher, aver: un rebord de $0 \mathrm{~m} .20$ à la base (pour supporter un grillage?) el un fond ronique dans lequel une fente laissait autrefois sourdre l'eau (fig. 16). De FIti. 16. - Coupe du fond du puils $n^{2} 2$. nombreux fragments de tuiles y furent recueillis. Signalons aussi: près de l'hòtel, un puisard utilisant la panse d'une large amphore romaine (fig. 17) qui a èté transportíe is l'école de Saint-Aubin.

\section{6) Le cimetière franc.}

Quand l'habitat eut été détruit (re siècle?). sans doute par le feu, si l'on en juge par les couches de charbons de bois et de cendres qui furent trouvées en divers endroits ot notamment en $A 5$, et $C$ a et dans la fosse $I I$. le site ne fut pas abandomé pour aulant. Il servit en tous as à de nombreux enterrements. Rappelons les découvertes de sareophages failes i diverses reprises sur le Camp Romain et spécialement à la coupure de la falaise. Pour notre purt, nous avous fouille +1 lombeaux.

Le cimetière occupe une surface l'environ 25 ares. Les lombes sont orientées est-ouest et disposées en 12 ou 13 rangées sans distinction entre les sépultures d'hommes, de femmes ou d'enfants. Nous avons reconnu 12 tombes d'enfanls ot 21 d'adultes; les autres cas sont indéterminés. Les sépultures se rencontraient a des profondeurs lrès variables constituant 
ainsi deux grandes séries, l'une d'une dizaine de tombes en surface, l'autre de deux douzaines en couche plus profonde. Les cercueils sont formés de pierres plates généralement brutes, calées avec des tessons, des coquilles, du silex ou des fragments de tuiles romaines. Le fond des tombeaux est dallé: on utilise le sommet d'une fondation antique, quelque pavage cimenté, ou même la simple glaise rouge bien nivelée. Les couvercles étaient

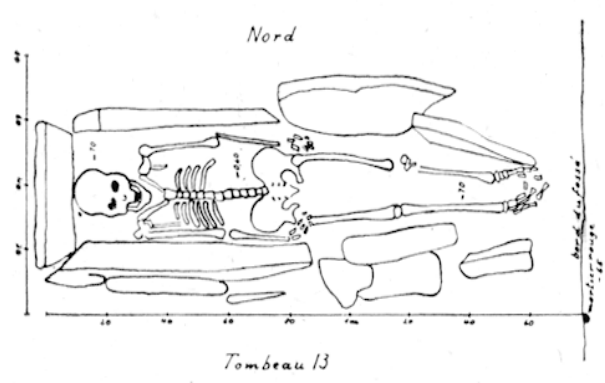

liki. Is. - Tomb: n" 1:3. formés de pierres plates qui furent rehouvées rarement in situ. Les squelettes, souvent bien conservés, ítaient couchés sur le dos et allongés, tête ver's l'ouest (fig. 18), les bras le plus souvent allongés sans que les mains fussent jamais croisées. Quelques (ombeaux contenaient beaucoup de ('harbons de bois (cercueil?). Aucun mobilier funciraire n'a été trouré. Les comparaisons, dójà indiquées par $\mathrm{M}$. Doranlo ${ }^{1}$, avee les cimetieres de Saint-l'rsin, Courseulles-sur-Mer, (6)lombières-sur-Seulles, etc., prouvent qu'il s'agit de tombes nérovingiennes assez récentes.

\section{Conclusions historiques.}

Si limités qu'aient done étri nos sondages, en raison même des circonstances, ils nous ont cependant fait connaître avec quelque précision historique un habitat principalement gallo-romain assez intéressant. Il resterait a identifier re qu'aucune dérouverte épigraphique n'est malheu. reusement venu faire. On sait que depuis longtemps les crudits songent à placer la la ville de Grammona, siture au Litus Suxonicum par la Notitia Dignitatum. Un fragment d'inscription trouvé à Bernières en 1940 confirmerait peut-être cette hỵpothèse, car on y lit:

\section{$\mathrm{I} \cdot \mathrm{OPT} \cdot \mathrm{Col}$ \\ RMOR $\cdot$ GR \\ $\mathrm{VA} \cdot \mathrm{LI} \cdot \mathrm{ATT}$}

Mais cette mention d'un optio de la cohorte armoricaine de $G R$ (ammona), décédé a l'âge de 51 ans, n'offre aurune garantie d'authenticité2 et il convient d'attendre les progrès des recherches archéologiques sur le site.

\section{Eugen EBLÉ.}

(1) Cf. Bull. Soc. Int. Norm., XLVIII, 1940, p. 544 sq.

(2) L'inscription nous a élé communiquée par II. Doranlo. Celle pierre lui alurait be montrée par le propriétaire de la villa "Grannona ", i Bernières, en 1940. On voil qu'il faut se métier, ainsi que le souligne M. Doranlo. 